\title{
Sensitive carbon monoxide detection based on light-induced thermoelastic spectroscopy with a fiber-coupled multipass cell [Invited]
}

\author{
Xiaonan Liu (刘晓楠) and Yufei Ma (马欲飞) * \\ National Key Laboratory of Science and Technology on Tunable Laser, Harbin Institute of Technology, Harbin 150001, China \\ *Corresponding author: mayufei@hit.edu.cn \\ Received December 5, 2021 | Accepted December 29, 2021 | Posted Online January 24, 2022
}

\begin{abstract}
A sensor based on light-induced thermoelastic spectroscopy (LITES) with a fiber-coupled multipass cell was demonstrated for carbon monoxide ( $\mathrm{CO}$ ) detection. The fiber-coupled structure has the merits of reducing optical interference and difficulty in optical alignment and increasing system robustness. A $1.57 \mu \mathrm{m}$ continuous wave distributed feedback diode laser was used as the excitation source. A minimum detection limit of 9 ppm was obtained, and the calculated normalized noise equivalent absorption coefficient was $1.15 \times 10^{-7} \mathrm{~cm}^{-1} \cdot \mathrm{W} \cdot \mathrm{Hz}^{-1 / 2}$. The reported $\mathrm{CO}$-LITES sensor showed excellent linear concentration response and system stability.
\end{abstract}

Keywords: light-induced thermoelastic spectroscopy; quartz tuning fork; CO concentration.

DOI: 10.3788/COL202220.031201

\section{Introduction}

Carbon monoxide (CO) is a colorless, odorless, and toxic gas that is harmful to humans. CO binds to hemoglobin in the blood, which in turn causes symptoms of oxygen deprivation in human tissues ${ }^{[1]}$. The main source of $\mathrm{CO}$ is the incomplete combustion of carbonaceous fuels. In addition to natural phenomena such as forest fires and volcanic eruptions, a large amount of $\mathrm{CO}$ is also generated by industrial processes and motor vehicle exhaust in urban areas. In recent years, the use of fossil fuels has gradually increased, making the $\mathrm{CO}$ pollution to atmosphere serious ${ }^{[2,3]}$. In addition, the detection of $\mathrm{CO}$ concentration exhaled from the human body can be used for clinical diagnosis ${ }^{[4,5]}$. Therefore, it is necessary to develop $\mathrm{CO}$ sensors with high sensitivity and real-time monitoring characteristics.

The quartz tuning fork (QTF) has become an attractive alternative to the photodetector by virtue of its high sensitivity, wide spectrum response band, small size, and strong noise immunity $^{[6-10]}$. QTF-based gas sensing technologies, quartzenhanced photoacoustic spectroscopy (QEPAS), and lightinduced thermoelastic spectroscopy (LITES), which were firstly, to the best of our knowledge, reported in 2002 and 2018 by Tittel $^{[11]}$ and $\mathrm{Ma}^{[12]}$, respectively, both use QTF as a detection unit. However, QEPAS requires QTF to be placed in the tested gas environment ${ }^{[13-20]}$, which means that it is a contact measurement method $^{[21]}$. This therefore limits its application in some specific areas such as combustion field diagnosis and corrosive gas detection, because the resonance characteristics of QTF will be affected in such conditions ${ }^{[22]}$.

LITES technology overcomes the above limitation well. The detection module can be placed far away from the environment to be tested, so that QTF will not be affected ${ }^{[23-27]}$. In LITES, after being absorbed by target gas, the laser is irradiated on the crystal surface of QTF. The light will be absorbed by the quartz crystal and converted into photothermal energy ${ }^{[28,29]}$. Due to the light-induced thermoelastic effect, QTF produces mechanical movement. When the laser modulation frequency is consistent with the resonance frequency of QTF, the mechanical vibration of QTF is enhanced, and the maximum value of the piezoelectric signal is obtained. It is worth emphasizing that the amount of change in laser intensity during this process determines the magnitude of the thermoelastic signal of QTF. Therefore, custom $\mathrm{QTF}^{[30]}$ and that covered with high absorption materials on QTF surface ${ }^{[31,32]}$ are usually adopted to enhance the thermoelastic effect. However, custom-made QTF and material coating are difficult to manufacture and have the increased experimental cost.

By installing concave mirrors with high reflectivity inside a multipass cell (MPC), multiple reflections of the light beam can be achieved to increase the optical absorption length in LITES. However, conventional MPC usually requires the light beam to be incident in a specific angle to achieve an ideal optical length, which significantly increases the difficulty of optical alignment and reduces the stability of the sensor system due to the usage of plenty of optical elements. In addition, the 
presence of optical windows can also make the system susceptible to interference phenomena ${ }^{[33]}$. In order to address the above problems, a fiber-coupled MPC was introduced in this manuscript. The optical path outside the MPC is transmitted by an optical fiber to eliminate the deviation of the optical path in traditional MPCs caused by destabilizing factors such as vibration. By using this fiber-coupled MPC, the optical interference was reduced, and the system had an easy optical adjustment. Furthermore, the coupled fiber with wide wavelength band of 1260-1675 nm was adopted, and the diode laser suitable for this wavelength band has the advantages of high efficiency, small size, and low $\operatorname{cost}^{[34,35]}$.

In this paper, a fiber-coupled MPC with an optical path length of $40 \mathrm{~m}$ is introduced to the LITES technique to increase the absorption length for the first time, to the best of our knowledge. The fiber-coupled structure has the merits of eliminating interference, reducing difficulty in optical alignment, and increasing system robustness. A diode laser with emission wavelength of $1.57 \mu \mathrm{m}$ was adopted as the excitation source. A commercial QTF with a quality (Q)-factor of 14,248 and a resonance frequency of $32.757 \mathrm{kHz}$ was chosen as the detector for lightinduced thermoelastic effect. $\mathrm{CO}$ was selected as the analysis gas to evaluate the detection performance of the sensing system because of its important application in combustion diagnosis, environmental monitoring, and medical diagnosis.

\section{Experimental Setup}

The simulation of the $\mathrm{CO}$ absorption line based on HITRAN 2012 database is shown in Fig. $1^{[36]}$. From Fig. 1(a), it can be found that $\mathrm{CO}$ absorption of the fundamental vibrationalrotational band ( $1 v$ band) is located at $4.7 \mu \mathrm{m}$. However, the quantum cascade laser covering this wavelength range is expensive and bulky ${ }^{[37,38]}$. Due to the difficulty and high cost of fiber coupling for lasers at $2.3 \mu \mathrm{m}$ ( $2 v$ overtone band), the laser with

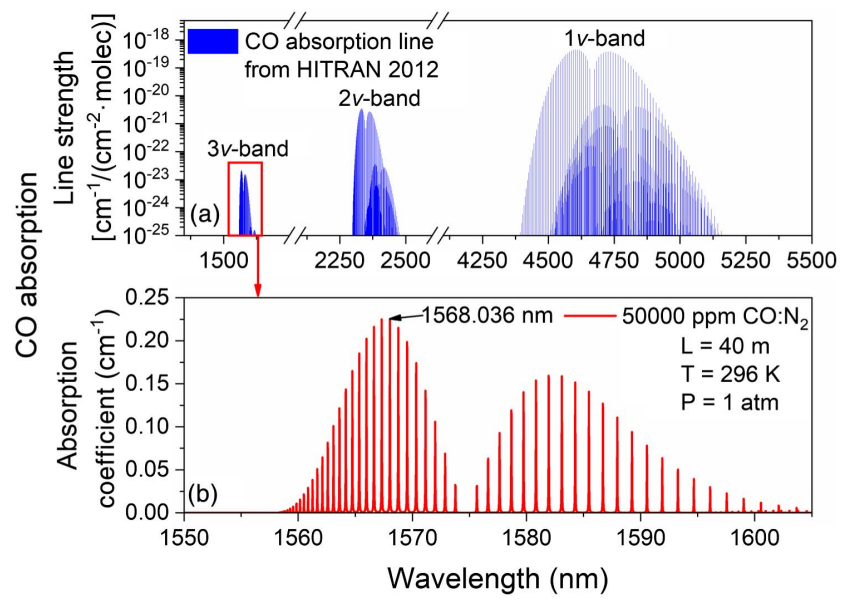

Fig. 1. Simulation of $\mathrm{CO}$ absorption spectra based on HITRAN 2012: (a) absorption line strength; (b) absorption coefficient of 50,000 ppm C0: $\mathrm{N}_{2}$ at $296 \mathrm{~K}$, $1 \mathrm{~atm}$, and $40 \mathrm{~m}$ optical path length. the emission wavelength of $1.5 \mu \mathrm{m}$ ( $3 v$ overtone band) was selected. As shown in Fig. 1(b), the spectral line with strong absorption at $1568.03 \mathrm{~nm}$ was chosen in this work.

The experimental configuration of the fiber-coupled MPCbased LITES sensor is shown in Fig. 2. A QTF with a resonance frequency $f_{0}$ of $32.768 \mathrm{kHz}$ (in vacuum) was selected, and its equivalent resistance was measured as $114 \mathrm{k} \Omega$. Wavelength modulation spectroscopy (WMS) with second harmonic ( $2 f$ ) detection was employed to reduce the noise level and simplify the data processing. A signal generator was used to generate a triangular wave with period of $1 \mathrm{~s}$ and duty cycle of $50 \%$ to scan across the absorption line. A lock-in amplifier was used to generate a sine wave signal with a frequency of $f_{0} / 2$. The triangle wave and sine wave were superimposed and sent to the laser controller. A distributed feedback (DFB) diode laser at $1.57 \mu \mathrm{m}$ was selected as the excitation source. The diode laser beam emission from the pigtail was firstly sent into the fiber-coupled MPC through a fiber connector. After passing through $40 \mathrm{~m}$ optical length in the MPC, the beam exited the MPC and was focused on the base of the QTF finger by a fiber collimator (FC). The divergence angle and focal length of the FC were $0.25^{\circ}$ and $30 \mathrm{~mm}$, respectively. The output power of the laser at the absorption line was $9.34 \mathrm{~mW}$; after passing through the MPC, the optical power decreased to $1.21 \mathrm{~mW}$. A beam $Q$ analyzer was used to capture the laser beam profile before and after passing through the MPC. The $2 \mathrm{D}$ beam profiles all showed Gaussian distribution. A piezoelectric signal was generated in QTF by the light-induced thermoelastic effect, which was further sent into the lock-in amplifier for demodulation and analysis. The bandwidth of the lock-in amplifier was set to $1.33 \mathrm{~Hz}$, and the filter roll off was $18 \mathrm{~dB}$ /oct. The integration time of the sensor was set to $60 \mathrm{~ms}$. A mass flow meter with an uncertainty of $3 \%$ was used to dilute the $50,000 \mathrm{ppm}$ (parts per million) $\mathrm{CO}: \mathrm{N}_{2}$ with pure nitrogen, and the flow rate was controlled at $300 \mathrm{~mL} / \mathrm{min}$.

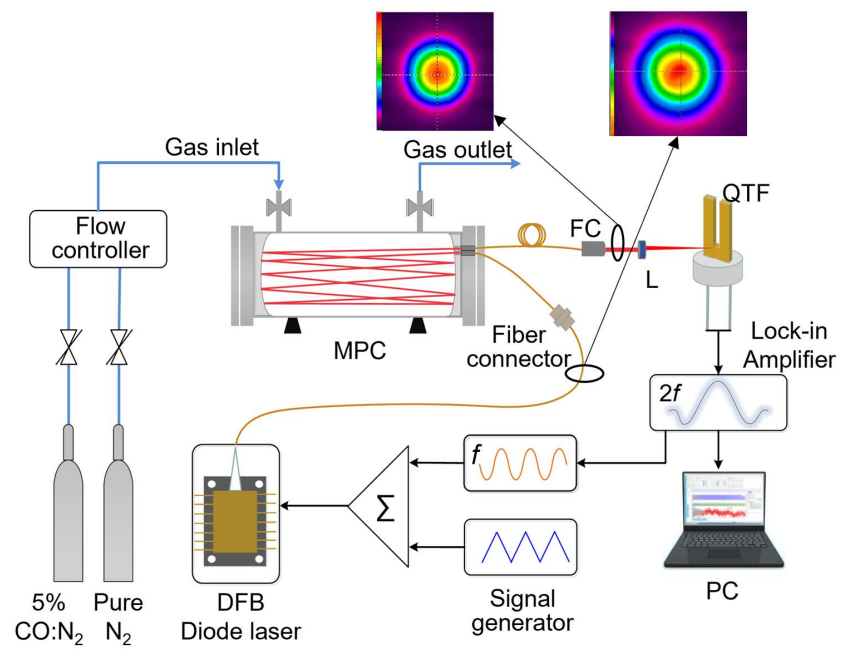

Fig. 2. Schematic diagram of CO-LITES sensing system. MPC, multipass cell; QTF, quartz tuning fork; $\Sigma$, adder; PC, personal computer; FC, fiber collimator; DFB, distributed feedback. 


\section{Results and Discussion}

Firstly, the resonance frequency $f_{0}$ of QTF at the experimental condition of atmospheric pressure was measured through the electrical excitation method. As is shown in Fig. 3, the $f_{0}$ and bandwidth $\Delta f$ were measured as $32.757 \mathrm{kHz}$ and $2.32 \mathrm{~Hz}$, respectively. The $Q$-factor was calculated as 14,248 according to the equation $Q=f_{0} / \Delta f$, indicating its excellent performance.

In order to get a strong response of the CO-LITES sensor, the laser wavelength modulation depth, an important parameter of the second harmonic method in WMS, should be optimized. Figure 4 shows the relationship between the normalized $2 f$ signal amplitude of the CO-LITES sensor and laser wavelength modulation depth. It can be found that the $2 f$ signal amplitude of the CO-LITES sensor increased firstly and then fell down with the increase of modulation depth. The maximum $2 f$ signal amplitude was obtained when the modulation depth was $0.55 \mathrm{~cm}^{-1}$. Therefore, in the following experiments the optimum modulation depth of $0.55 \mathrm{~cm}^{-1}$ was adopted.

To investigate the linear response of the CO-LITES sensor to $\mathrm{CO}$ concentration, the $2 f$ signals of the sensor were collected at different concentrations. After $5 \% \mathrm{CO}: \mathrm{N}_{2}$ was diluted by pure nitrogen $\left(\mathrm{N}_{2}\right)$ to $1000,2000,5000,10,000$, and $20,000 \mathrm{ppm}$ in proportion, the gas was fed to the MPC at a constant flow rate. The measured values of the $2 f$ signal at different concentrations are shown in Fig. 5. The obtained $2 f$ signal amplitude as a function of $\mathrm{CO}$ concentration is depicted in Fig. 6. The calculated $\mathrm{R}$-square value of a linear fit of the $2 f$ signal amplitude was equal to $\sim 0.99$, indicating that the reported CO-LITES sensor has a good linear response to the $\mathrm{CO}$ concentrations.

Finally, to further investigate the optimal detection performance and system stability of the fiber-coupled MPC-based CO-LITES sensor, the signal was monitored in real time of $2 \mathrm{~h}$ under the condition of pure $\mathrm{N}_{2}$ in the MPC. The obtained data were subjected to Allan deviation analysis, and the result is shown in Fig. 7. The minimum detection limit (MDL)

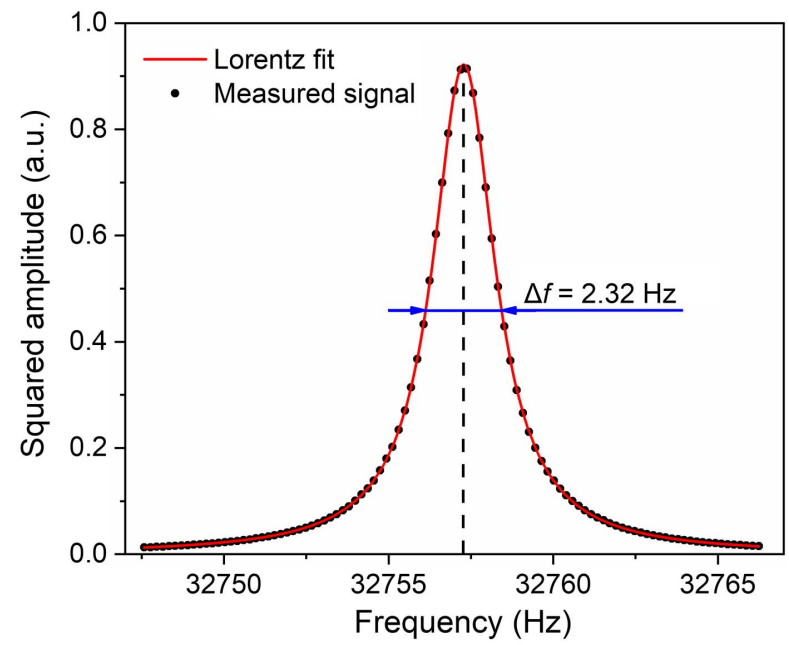

Fig. 3. Normalized and squared amplitude of QTF response as a function of frequency.

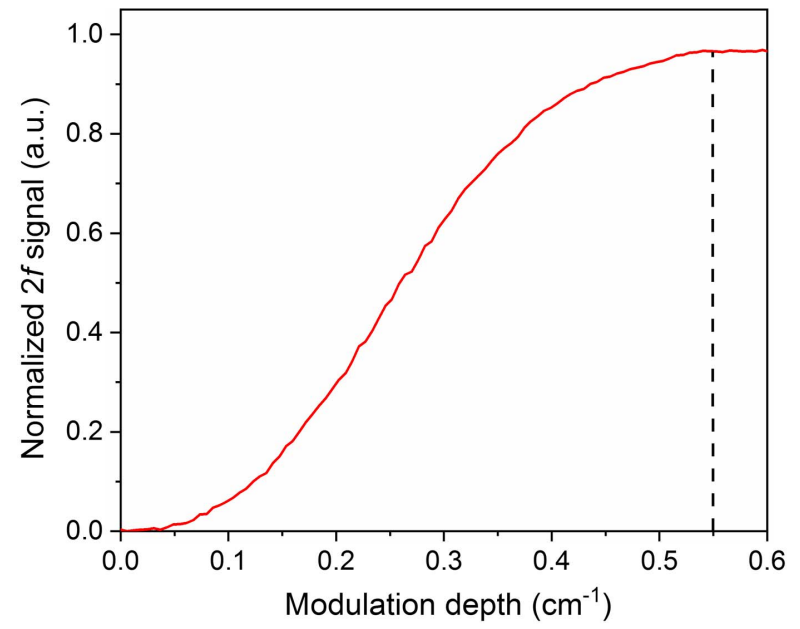

Fig. 4. Normalized $2 f$ signal amplitude of CO-LITES sensor as a function of laser wavelength modulation depth.

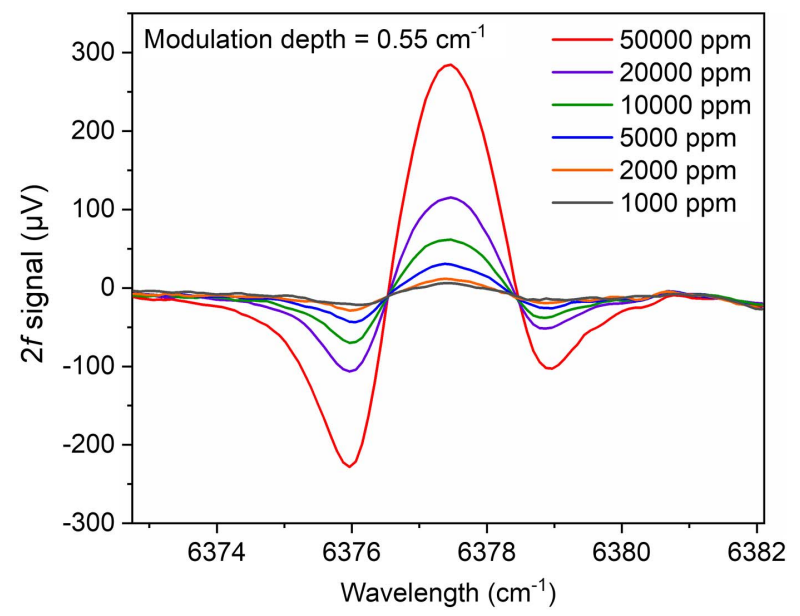

Fig. 5. CO-LITES sensor $2 f$ signal for different concentrations.

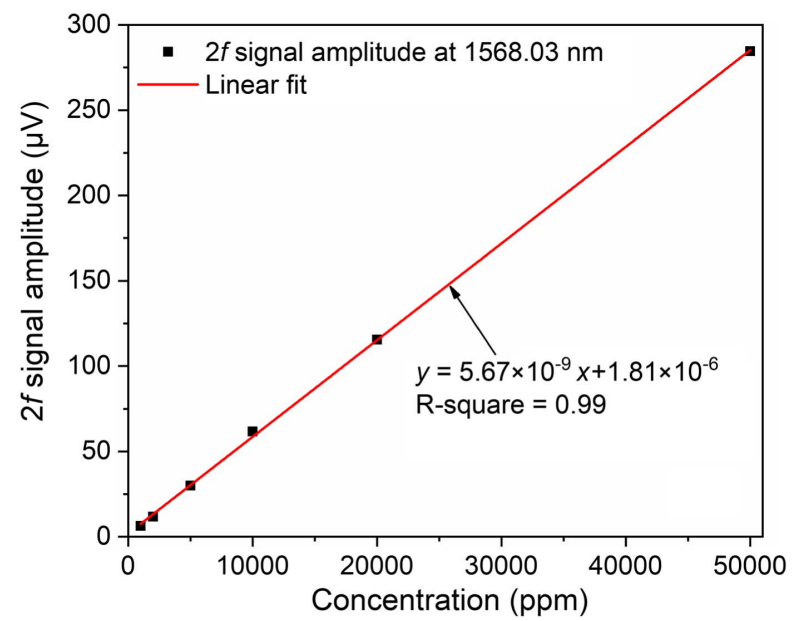

Fig. 6. Linear relationship between the peak value of the $2 f$ signal and CO concentration. 


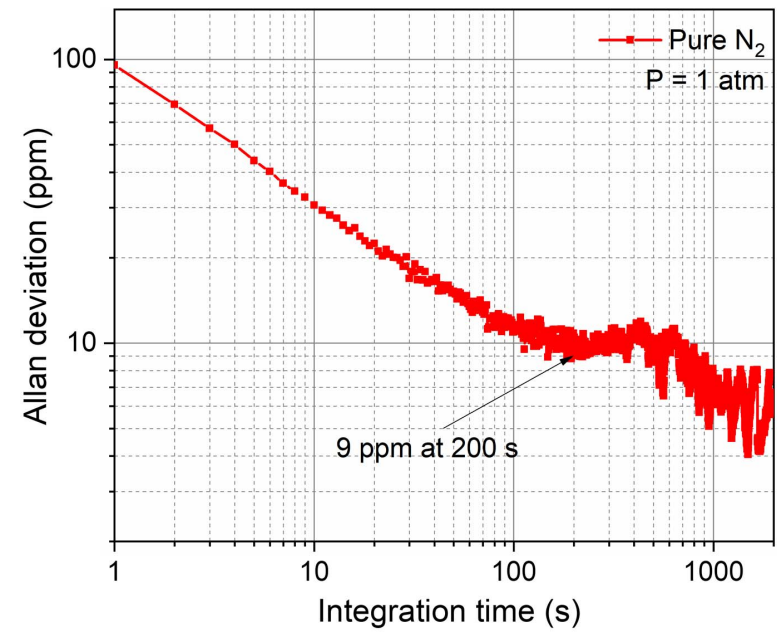

Fig. 7. Allan deviation analysis of the CO-LITES sensor.

Table 1. Performance Comparison of Two QTF-Based Methods for CO Detection.

\begin{tabular}{lc}
\hline Method & NNEA $\left(\mathrm{cm}^{-1} \cdot \mathrm{W} \cdot \mathrm{Hz}^{-1 / 2}\right]$ \\
\hline QEPAS $^{[39]}$ & $1.45 \times 10^{-5}$ \\
This work & $1.15 \times 10^{-7}$ \\
\hline
\end{tabular}

of the CO-LITES sensor was $96 \mathrm{ppm}$ at an integration time of $1 \mathrm{~s}$. When the integration time was extended to $200 \mathrm{~s}$, the MDL was improved to $9 \mathrm{ppm}$.

In addition, the normalized noise equivalent absorption coefficient (NNEA) was also used to evaluate the sensitivity of the sensor. It can be expressed as follows ${ }^{[22]}$ :

$$
\mathrm{NNEA}=\frac{\alpha P}{\Delta f},
$$

where $\alpha$ is the absorption coefficient, $P$ is the laser power at the absorption line, and $\Delta f$ is the detection bandwidth of the lockin amplifier. The calculated NNEA for this fiber-coupled MPCbased CO-LITES sensor was $1.15 \times 10^{-7} \mathrm{~cm}^{-1} \cdot \mathrm{W} \cdot \mathrm{Hz}^{-1 / 2}$. The performance of this sensor was compared to the other QTF-based gas sensor of QEPAS. The comparisons are listed in Table 1. It can be found that NNEA for the CO-QEPAS sensor is $1.45 \times 10^{-5} \mathrm{~cm}^{-1} \cdot \mathrm{W} \cdot \mathrm{Hz}^{-1 / 2}$. The detection performance achieved by this fiber-coupled MPC-based CO-LITES sensor was improved significantly.

\section{Conclusions}

In summary, a novel CO-LITES sensor based on a fiber-coupled MPC was presented for the first time, to the best of our knowledge. The fiber-coupled MPC with an effective optical path of $40 \mathrm{~m}$ was used to increase $\mathrm{CO}$ absorption and finally to enhance the light-induced thermoelastic effect in QTF. A pigtailed, near infrared, DFB diode laser emitting at $1.57 \mu \mathrm{m}$ was selected as the laser emission source. The laser wavelength modulation depth for the selected absorption line at $1568.03 \mathrm{~nm}$ was optimized to $0.55 \mathrm{~cm}^{-1}$. An MDL of $9 \mathrm{ppm}$ was obtained at an integration time of $200 \mathrm{~s}$ for this sensor, and the calculated NNEA was $1.15 \times 10^{-7} \mathrm{~cm}^{-1} \cdot \mathrm{W} \cdot \mathrm{Hz}^{-1 / 2}$. The CO-LITES sensor showed excellent linear response for different concentrations of $\mathrm{CO}$ gas. Due to the fiber-coupled feature of excellent stability and minimization to optical interference, this CO-LITES sensor is suitable for industrial measurements and environmental monitoring.

\section{Acknowledgement}

This work was supported by the National Natural Science Foundation of China (Nos. 62022032, 61875047, and 61505041), Natural Science Foundation of Heilongjiang Province of China (No. YQ2019F006), Fundamental Research Funds for the Central Universities, and Financial Grant from the Heilongjiang Province Postdoctoral Foundation (No. LBH-Q18052).

\section{References}

1. L. D. Prockop and R. I. Chichkova, "Carbon monoxide intoxication: an updated review," J. Neurol. Sci. 262, 122 (2007).

2. M. A. Khalil and R. A. Rasmussen, "Carbon monoxide in the earth's atmosphere: increasing trend," Science 224, 54 (1984).

3. H. Golmohamadi, R. Keypour, and P. Mirzazade, "Multi-objective cooptimization of power and heat in urban areas considering local air pollution,” Eng. Sci. Technol. 24, 372 (2021).

4. W. Q. Cao and Y. X. Duan, "Breath analysis: potential for clinical diagnosis and exposure assessment," Clin. Chem. 52, 800 (2006).

5. G. Lippi, G. Rastelli, T. Meschi, L. Borghi, and G. Cervellin, "Pathophysiology, clinics, diagnosis and treatment of heart involvement in carbon monoxide poisoning," Clin. Biochem. 45, 1278 (2012).

6. Y. F. Ma, Y. Tong, Y. He, X. G. Jin, and F. K. Tittel, “Compact and sensitive mid-infrared all-fiber quartz-enhanced photo-acoustic spectroscopy sensor for carbon monoxide detection," Opt. Express 27, 9302 (2019).

7. Y. He, Y. F. Ma, Y. Tong, X. Yu, and F. K. Tittel, “A portable gas sensor for sensitive CO detection based on quartz-enhanced photoacoustic spectroscopy," Opt. Laser Technol. 115, 129 (2019).

8. Q. D. Zhang, J. Chang, Z. H. Cong, and Z. L. Wang, "Application of quartz tuning fork in photodetector based on photothermal effect," IEEE Photonic. Tech. Lett. 31, 1592 (2019).

9. Z. T. Lang, S. D. Qiao, Y. He, and Y. F. Ma, "Quartz tuning fork-based demodulation of an acoustic signal induced by photo-thermo-elastic energy conversion," Photoacoustics 22, 100272 (2021).

10. S. D. Qiao, Y. He, and Y. F. Ma, “Trace gas sensing based on single-quartzenhanced photoacoustic-photothermal dual spectroscopy," Opt. Lett. 46, 2449 (2021).

11. A. A. Kosterev, Y. A. Bakhirkin, R. F. Curl, and F. K. Tittel, "Quartz-enhanced photoacoustic spectroscopy,” Opt. Lett. 27, 1902 (2002).

12. Y. F. Ma, Y. He, Y. Tong, X. Yu, and F. K. Tittel, "Quartz-tuning-fork enhanced photothermal spectroscopy for ultra-high sensitive trace gas detection," Opt. Express 26, 32103 (2018).

13. A. Sampaolo, P. Patimisco, L. Dong, A. Geras, G. Scamarcio, T. Starecki, F. K. Tittel, and V. Spagnolo, "Quartz-enhanced photoacoustic spectroscopy exploiting tuning fork overtone modes," Appl. Phys. Lett. 107, 231102 (2015).

14. H. Wu, L. Dong, H. Zheng, Y. Yu, W. Ma, L. Zhang, W. Yin, L. Xiao, S. Jia, and F. K. Tittel, "Beat frequency quartz-enhanced photoacoustic spectroscopy for fast and calibration-free continuous trace-gas monitoring," Nat. Commun. 8, 15331 (2017). 
15. T. N. Ba, M. Triki, G. Desbrosses, and A. Vicet, "Quartz-enhanced photoacoustic spectroscopy sensor for ethylene detection with a $3.32 \mu \mathrm{m}$ distributed feedback laser diode," Rev. Sci. Instrum. 86, 02311 (2015).

16. K. Liu, X. Y. Guo, H. M. Yi, W. D. Chen, W. J. Zhang, and X. M. Gao, "Offbeam quartz-enhanced photoacoustic spectro-scopy," Opt. Lett. 34, 1594 (2009).

17. P. Patimisco, A. Sampaolo, L. Dong, F. K. Tittel, and V. Spagnolo, "Recent advances in quartz enhanced photoacoustic sensing," Appl. Phys. Rev. 5, 011106 (2018).

18. Y. F. Ma, R. Lewicki, M. Razeghi, and F. K. Tittel, "QEPAS based ppb-level detection of $\mathrm{CO}$ and $\mathrm{N}_{2} \mathrm{O}$ using a high power CW DFB-QCL," Opt. Express 21, 1008 (2013).

19. S. D. Qiao, Y. F. Ma, P. Patimisco, A. Sampaolo, Y. He, Z. T. Lang, F. K. Tittel, and V. Spagnolo, "Multi-pass quartz-enhanced photoacoustic spectroscopybased trace gas sensing," Opt. Lett. 46, 977 (2021).

20. H. D. Zheng, Y. H. Liu, H. Y. Lin, B. Liu, X. H. Gu, D. Q. Li, B. C. Huang, Y. C. Wu, L. P. Dong, W. G. Zhu, J. Y. Tang, H. Y. Guan, H. H. Liu, Y. C. Zhong, J. B. Fang, Y. H. Luo, J. Zhang, J. H. Yu, Z. Chen, and F. K. Tittel, "Quartz-enhanced photoacoustic spectroscopy employing pilot line manufactured custom tuning forks," Photoacoustics 17, 100158 (2020).

21. Y. F. Ma, Y. H. Hong, S. D. Qiao, Z. T. Lang, and X. N. Liu, "H-shaped acoustic micro-resonator based quartz-enhanced photoacoustic spectroscopy," Opt. Lett. (2022).

22. Y. F. Ma, Y. He, X. Yu, C. Chen, R. Sun, and F. K. Tittel, "HCl ppb-level detection based on QEPAS sensor using a low resonance frequency quartz tuning fork," Sens. Actuators B 233, 388 (2016).

23. T. T. Wei, A. Zifarelli, S. D. Russo, H. P. Wu, G. Menduni, P. Patimisco, A. Sampaolo, V. Spagnolo, and L. Dong, "High and flat spectral responsivity of quartz tuning fork used as infrared photodetector in tunable diode laser spectroscopy,” Appl. Phys. Rev. 8, 041409 (2021).

24. Y. He, Y. F. Ma, Y. Tong, X. Yu, and F. K. Tittel, "Ultra-high sensitive lightinduced thermoelastic spectroscopy sensor with a high $Q$-factor quartz tuning fork and a multipass cell," Opt. Lett. 44, 1904 (2019).

25. L. Hu, C. T. Zheng, M. H. Zhang, K. Y. Zheng, J. Zheng, Z. Song, X. Li, Y. Zhang, Y. D. Wang, and F. K. Tittel, "Long-distance in-situ methane detection using near-infrared light-induced thermo-elastic spectroscopy," Photoacoustics 21, 100230 (2021).

26. S. D. Qiao, Y. F. Ma, Y. He, P. Patimisco, A. Sampaolo, and V. Spagnolo, "Ppt level carbon monoxide detection based on light-induced thermoelastic spectroscopy exploring custom quartz tuning forks and a mid-infrared QCL," Opt. Express 29, 25100 (2021).

27. S. D. Russo, A. Zifarelli, P. Patimisco, A. Sampaolo, T. T. Wei, H. P. Wu, L. Dong, and V. Spagnolo, "Light-induced thermo-elastic effect in quartz tuning forks exploited as a photodetector in gas absorption spectroscopy," Opt. Express 28, 19074 (2020).

28. Y. Q. Hu, S. D. Qiao, Y. He, Z. T. Lang, and Y. F. Ma, "Quartz-enhanced photoacoustic-photothermal spectroscopy for trace gas sensing," Opt. Express 29, 5121 (2021).
29. X. N. Liu, S. D. Qiao, and Y. F. Ma, "Highly sensitive methane detection based on light-induced thermoelastic spectroscopy with a $2.33 \mu \mathrm{m}$ diode laser and adaptive Savitzky-Golay filtering," Opt. Express 30, 1304 (2022).

30. Y. F. Ma, Y. He, P. Patimisco, A. Sampaolo, S. D. Qiao, X. Yu, F. K. Tittel, and V. Spagnolo, "Ultra-high sensitive trace gas detection based on light-induced thermoelastic spectroscopy and a custom quartz tuning fork," Appl. Phys. Lett. 116, 011103 (2020).

31. C. Lou, X. Yang, X. Li, H. Chen, C. Chang, and X. Liu, "Graphene-enhanced quartz tuning fork for laser-induced thermoelastic spectroscopy," IEEE Sens. J. 21, 9819 (2021).

32. C. G. Lou, H. J. Chen, X. T. Li, X. Yang, Y. Zhang, J. Q. Yao, Y. F. Ma, C. Chang, and X. L. Liu, "Graphene oxide and polydimethylsiloxane coated quartz tuning fork for improved sensitive near-and mid-infrared detection," Opt. Express 29, 20190 (2021).

33. T. Y. Zhang, J. W. Kang, D. Z. Meng, H. Wang, Z. M. Mu, M. Zhou, and C. Chen, "Mathematical methods and algorithms for improving nearinfrared tunable diode-laser absorption spectroscopy," Sensors 18, 4295 (2018).

34. Y. F. Ma, Y. Q. Hu, S. D. Qiao, Y. He, and F. K. Tittel, “Trace gas sensing based on multi-quartz-enhanced photothermal spectroscopy," Photoacoustics 20, 100206 (2020).

35. X. Yin, L. Dong, H. Zheng, X. Liu, H. Wu, Y. Yang, W. Ma, L. Zhang, W. Yin, and L. Xiao, "Impact of humidity on quartz-enhanced photoacoustic spectroscopy based $\mathrm{CO}$ detection using a near-IR telecommunication diode laser," Sensors 16, 162 (2016).

36. I. E. Gordon, L. S. Rothman, C. Hill, R. V. Kochanov, Y. Tan, P. F. Bernath, M. Birk, V. Boudon, A. Campargue, K. V. Chance, B. J. Drouin, J. M. Flaud, R. R. Gamache, J. T. Hodges, D. Jacquemart, V. I. Perevalov, A. Perrin, K. P. Shine, M. A. H. Smith, J. Tennyson, G. C. Toon, H. Tran, V. G. Tyuterev, A. Barbe, A. G. Császár, V. M. Devi, T. Furtenbacher, J. J. Harrison, J.-M. Hartmann, A. Jolly, T. J. Johnson, T. Karman, I. Kleiner, A. A. Kyuberis, J. Loos, O. M. Lyulin, S. T. Massie, S. N. Mikhailenko, N. Moazzen-Ahmadi, H. S. P. Müller, O. V. Naumenko, A. V. Nikitin, O. L. Polyansky, M. Rey, M. Rotger, S. W. Sharpe, K. Sung, E. Starikova, S. A. Tashkun, J. V. Auwera, G. Wagner, J. Wilzewski, P. Wcisło, S. Yu, and E. J. Zak, "The HITRAN 2016 molecular spectroscopic database," J. Quant. Spectrosc. Radiat. Transf. 203, 3 (2017).

37. J. P. Waclawek, H. Moser, and B. Lendl, "Compact quantum cascade laser based quartz-enhanced photoacoustic spectroscopy sensor system for detection of carbon disulfide," Opt. Express 24, 6559 (2016).

38. W. Ren, A. Farooq, D. F. Davidson, and R. K. Hanson, "CO concentration and temperature sensor for combustion gases using quantum-cascade laser absorption near 4.7 mm," Appl. Phys. B 107, 849 (2012).

39. Y. F. Ma, Y. Guang, J. B. Zhang, X. Yu, and R. Sun, "Sensitive detection of carbon monoxide based on a QEPAS sensor with a $2.3 \mu \mathrm{m}$ fiber-coupled antimonide diode laser," J. Optics 17, 055401 (2015). 